\title{
Plasma Polymerization of Amines and Acetone: Functionalized Surfaces for Microarray Applications
}

\author{
Heinrich Jehle, ${ }^{1^{*}}$ Wolfgang Kranewitter ${ }^{2}$ \\ ${ }^{1}$ Greiner Bio-One GmbH, Maybachstrasse 2, D-72636 Frickenhausen, Tel.: +49 (0) \\ 7022/948426; Fax: +49 (0) 7022/948580; E-mail: heinrich.jehle@gbo.com \\ 2 Lambda GmbH, Industriestraße 6, A-4240 Freistadt
}

(Received: 10 October, 2006; published: 18 January, 2007)

\begin{abstract}
Plasma polymerization (PP) afforded amine as well as carbonyl containing films which are suitable for use in microarray analysis. The polymerization process with acetone (AC), ethylenediamine (EDA) and diethylamine (DEA) has to be performed in the presence of argon as a carrier gas to be successful. Different activation steps of the polymer based support yield surfaces which differ significantly in their elemental composition after the PP step. Angle dependent XPS proves a take off angle dependence of the carbonyl PP film in contrast to a homogeneous PP film for amine functionalized PP layers while microarray experiments have been successful for both functional films.

Keywords: Plasma Polymerization / XPS / Microarray analysis
\end{abstract}

\section{Introduction}

Microarray technology is an emerging and evolving science which had its origins in the last decade of the $20^{\text {th }}$ century [1-3]. In recent years, many applications of this pathway have attracted tremendous interest of lot of researchers with respect to fields like gene discovery, clinical diagnostics, pharmagenomics as well as toxicogenomics [4-6].

The basic setup of a microarray platform often consists of a solid support such as glass, polymer or tantal oxide and a surface chemistry which is capable of immobilizing biomolecules via ionic or covalent reaction pathways [8-9]. In general suitable surfaces are obtained via organosilane based condensation reactions [10] or photo chemically induced functionalization with unsaturated organic monomers [11]. The work presented here describes a novel pathway for the generation of surfaces which can be used for microarray analysis.

Plasma polymers can be described as thin polymeric films which are generated from corresponding monomers via continuous wave or pulsed radio frequency induced plasmas [12-13]. This process of formation of polymers involving the transformation of organic vapours often leads to unique characteristics of the films and therefore gives the opportunity for widespread applications [14-15].

The preservation of the functional group of the monomers is an indispensable need for the success of the use of plasma polymers for microarray analysis. Short et. al. exemplified a relationship between the monomer flow rate and the plasma power for unsaturated monomers such as acrylic acid and allyl alcohol in recent years [16]. Therefore, we examined relationships of several plasma deposition parameters such as surface activation, monomer flow rate, plasma power as well the effect of carrier gases with respect to monomer retention of precursors which do not contain carbon- 
carbon double bonds. Additionally the amine and carbonyl containing films resulting from plasma polymerization processes using several amines and acetone have to be stable for a longer period of time to be a useful tool in microarray applications.

\section{Results and discussion:}

The polymerization process of ethylendiamine (EDA), diethylamine (DEA) and acetone (AC) has afforded PP (plasma polymer) surfaces with controlled concentrations of amine and carbonyl moieties. Acetone as a precursor was chosen to conceive a pathway for the generation of ketone containing films which are as suitable for microarray analysis as customary used aldehyde surfaces commonly prepared via SOL GEL methods. EDA and DEA as primary and secondary amines have been studied with respect to the comparison of the nitrogen content of the resulting functionalized surfaces.

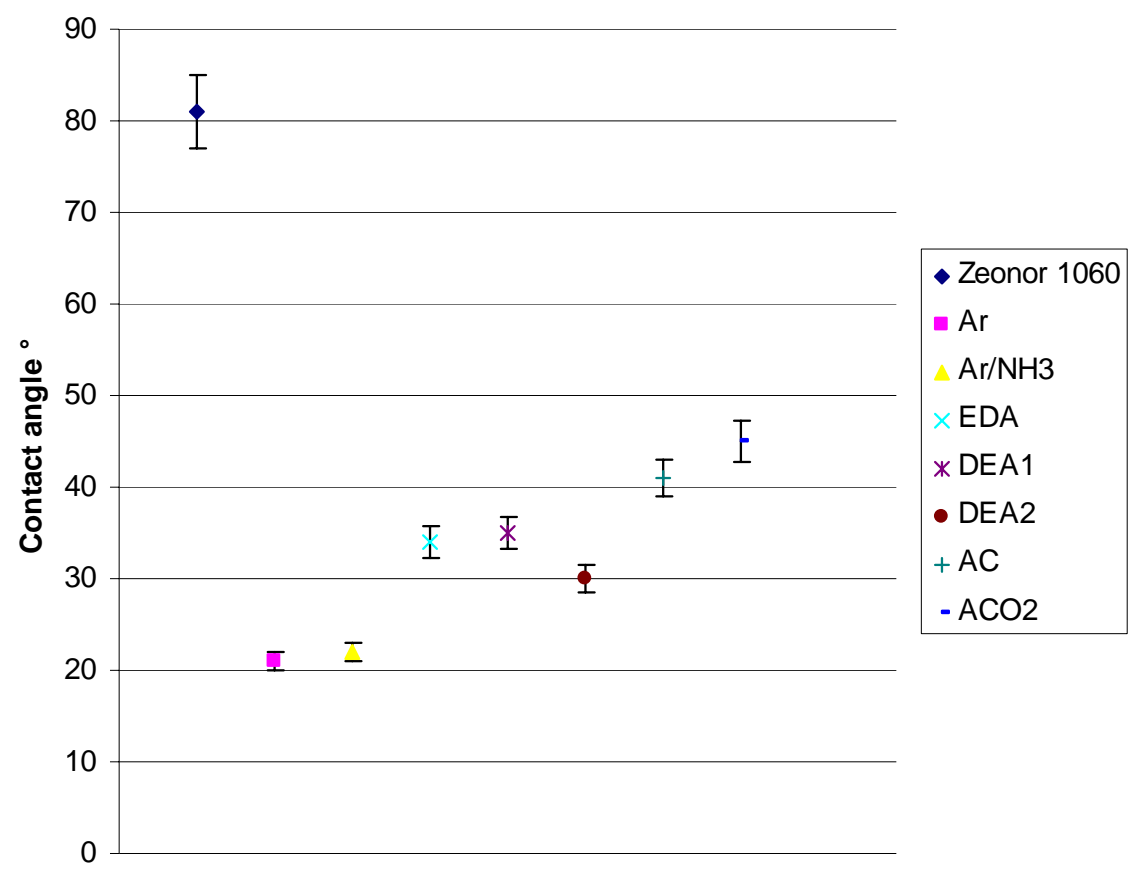

Fig. 1. Water contact angles of untreated, activated and polymerized slides.

Contact angle measurements (Fig. 1) can show that the surface energy highly depends on the monomer source and that contact angles change to higher values upon polymerization processes.

Contact angles of carbonyl surfaces (AC/ACO2) are higher compared to the amine surfaces but are still in a reasonable range to yield satisfying spot sizes in the printing process of the microarray experiment.

The values of the nitrogen peaks as well as the slight broadening give indication of the existence of several nitrogen containing species in the PP, if EDA or DEA are used as precursors. The existence of primary amines is proved by the fluorescamin assay [17] with an excitation wavelength of 380nm and the detection at $470 \mathrm{~nm}$. The elemental concentrations obtained from angle dependent XPS display the independence from the take off angle and therefore prove the existence of a 
homogeneous film with a thickness of minimum $4 \mathrm{~nm}$. Compared to this finding conventional glass slides which are functionalized with 3-aminopropyltrimethoxysilane via acid assisted condensation reactions [18] prove to have approximately 1.8 $\mathrm{nm}$ thick functional layers with an nitrogen content of 2.3 to $2.7 \%$.

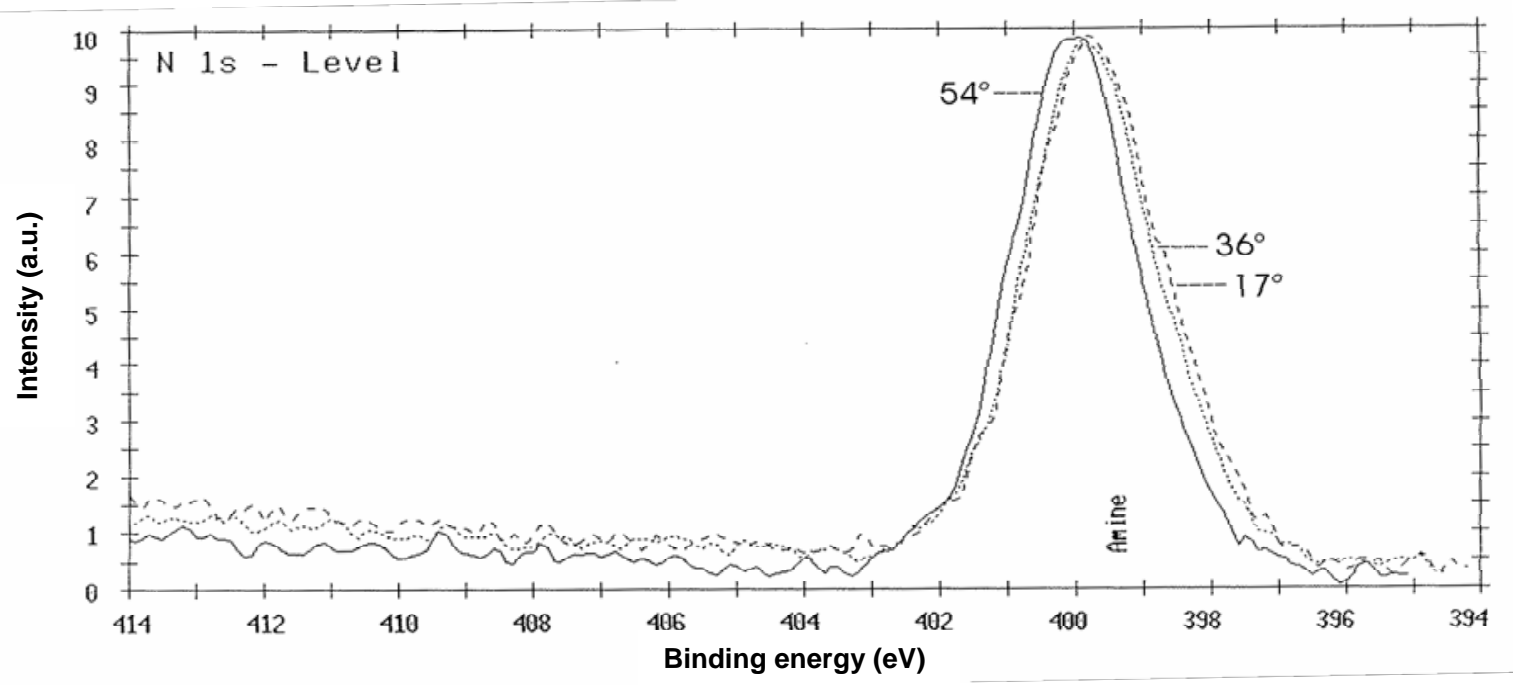

Fig. 2. Angle dependent XPS $N_{1 s}$ spectra of the series EDA.

A dependence of the nitrogen content from the activation procedure is observed by the comparison of series DEA1 with an $\mathrm{Ar} / \mathrm{NH}_{3}$ activation step with series DEA2 with an argon activation with high amounts of nitrogen moieties of up to $25 \%$ (DEA1) in contrast to $14 \%$ nitrogen containing functional groups in the series DEA2 (Fig. 3).

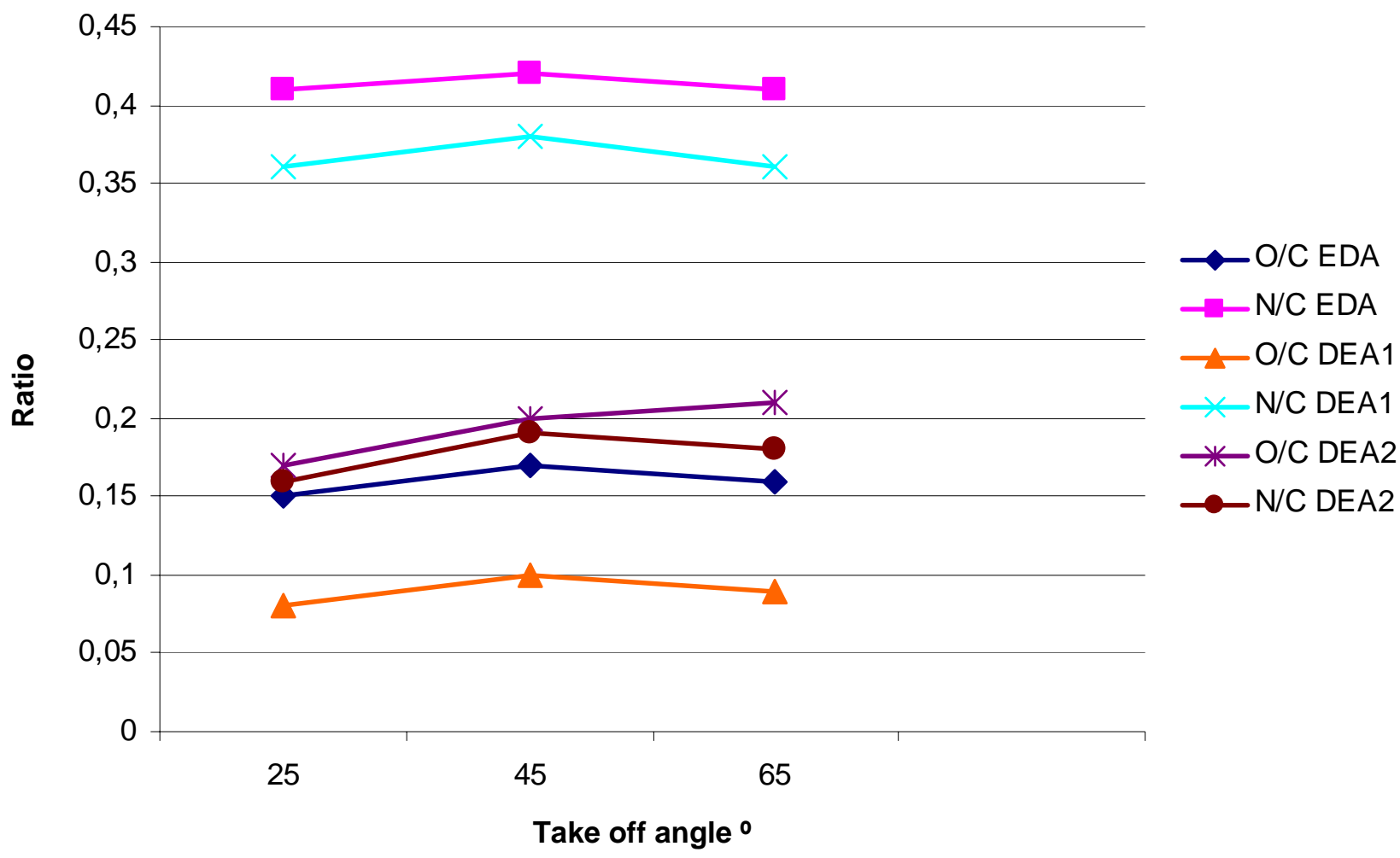

Fig. 3. Angle dependent carbon (C), oxygen (O) and nitrogen (N) ratios of PP films. 
The oxygen uptake can be deduced by the XPS data and can not be prevented even by $60 \mathrm{~min}$ argon loading of the plasma reactor after completion of the PP reaction.

Ageing of the DEA1 series for $170 \mathrm{~h}$ at $50{ }^{\circ} \mathrm{C}$ followed by XPS analysis yielded surfaces which do not differ significantly from the original PP films with respect to elemental composition as well as the contact angles.

For the generation of carbonyl functionalized PP films the activation of the polymer based support is performed via argon / oxygen treatment. The plasma polymerization of acetone in the presence of argon (AC) or argon and oxygen (ACO2) as carrier gases yields similar surfaces without an effect on the elemental composition (Fig. 4).

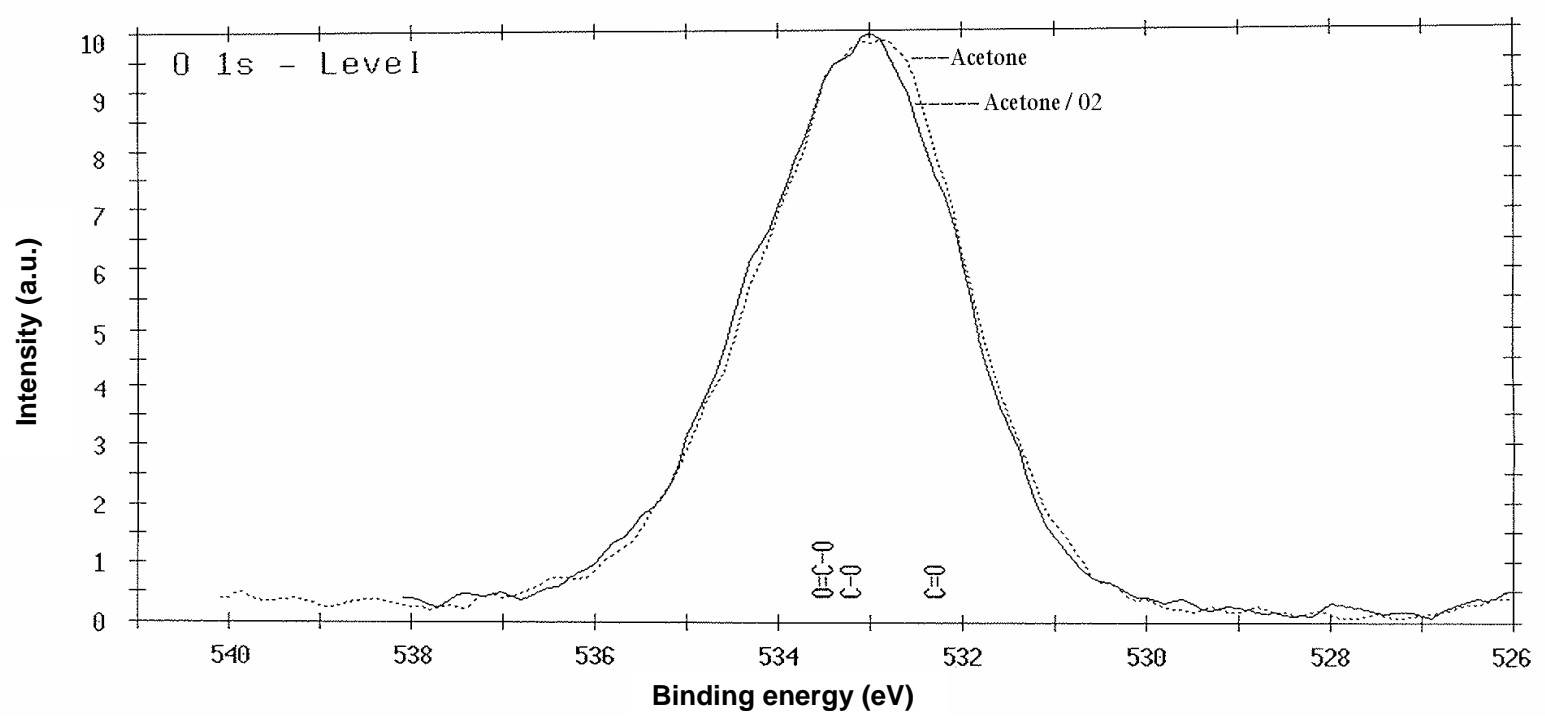

Fig. 4. $X P S \mathrm{O}_{1 \mathrm{~s}}$ spectra series $\mathrm{AC} / \mathrm{ACO}$.

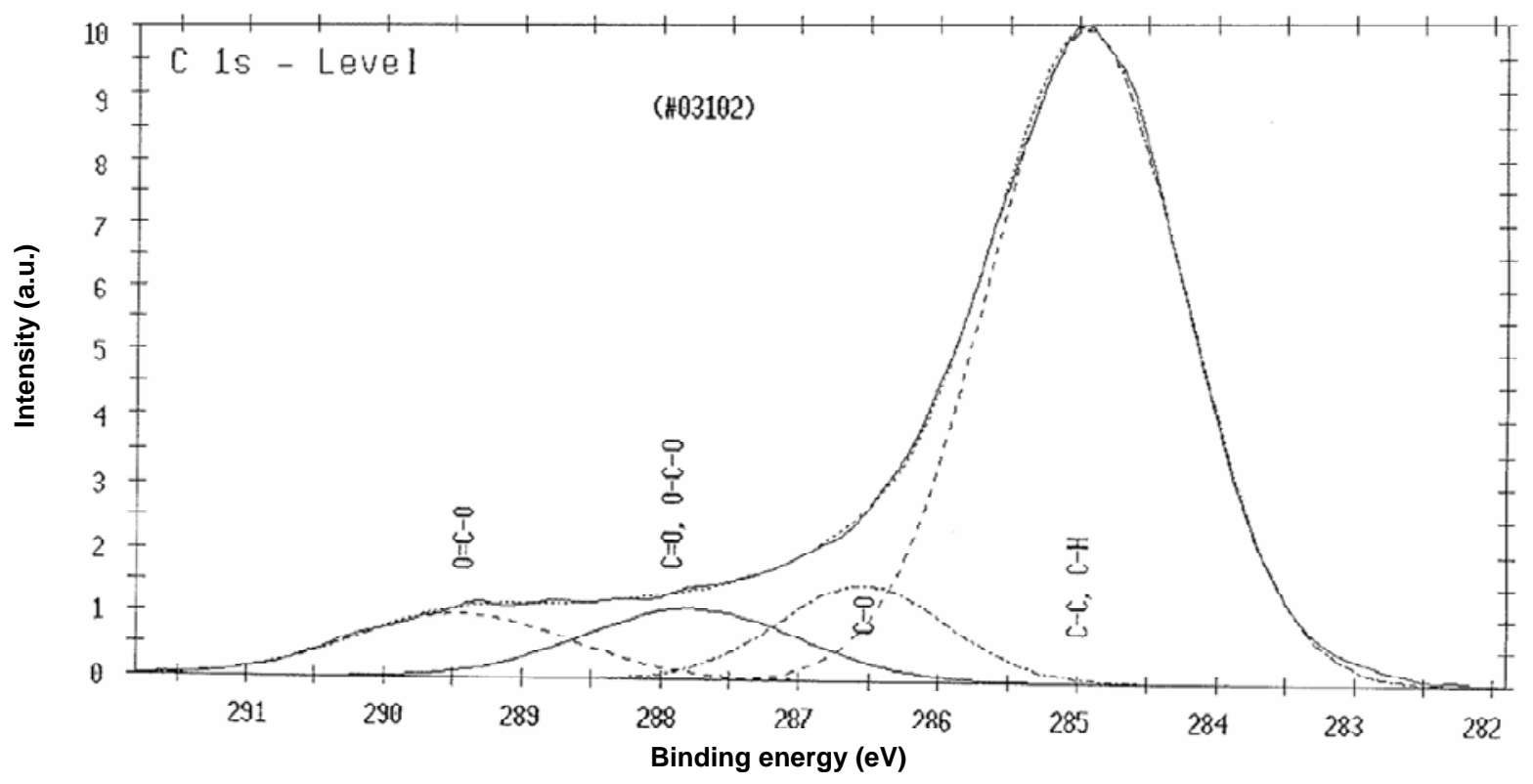

Fig. 5: $\mathrm{XPS} \mathrm{C}_{1 \mathrm{~s}}$ spectra series ACO2. 
The comparison of the carbon spectra of the series AC and ACO2 exemplifies the concurrence of the oxygen containing surfaces. The existence of carbonyl species is exemplified via reductive amination with 1,4-daminobutane and $\mathrm{NaBH}_{3} \mathrm{CN}$ in ethanol and the fluorescamine assay. A detailed examination of the existing carbon bonding modes of the $\mathrm{ACO} 2$ series via incorporation of compensation curves results in an estimation of C-C; C-H 74\%; C-O 8\%, O-C-O / C=O 10\%, O-C=O 8\% (Fig. 5).

In contrast to the amino functionalized PP films, significant angle dependence in the elemental composition of the carbonyl derivatives is observed with a decreasing share of oxygen in higher take off angles which indicate that the maximum layer thickness can be estimated as $3.5 \mathrm{~nm}$ (Fig. 6).

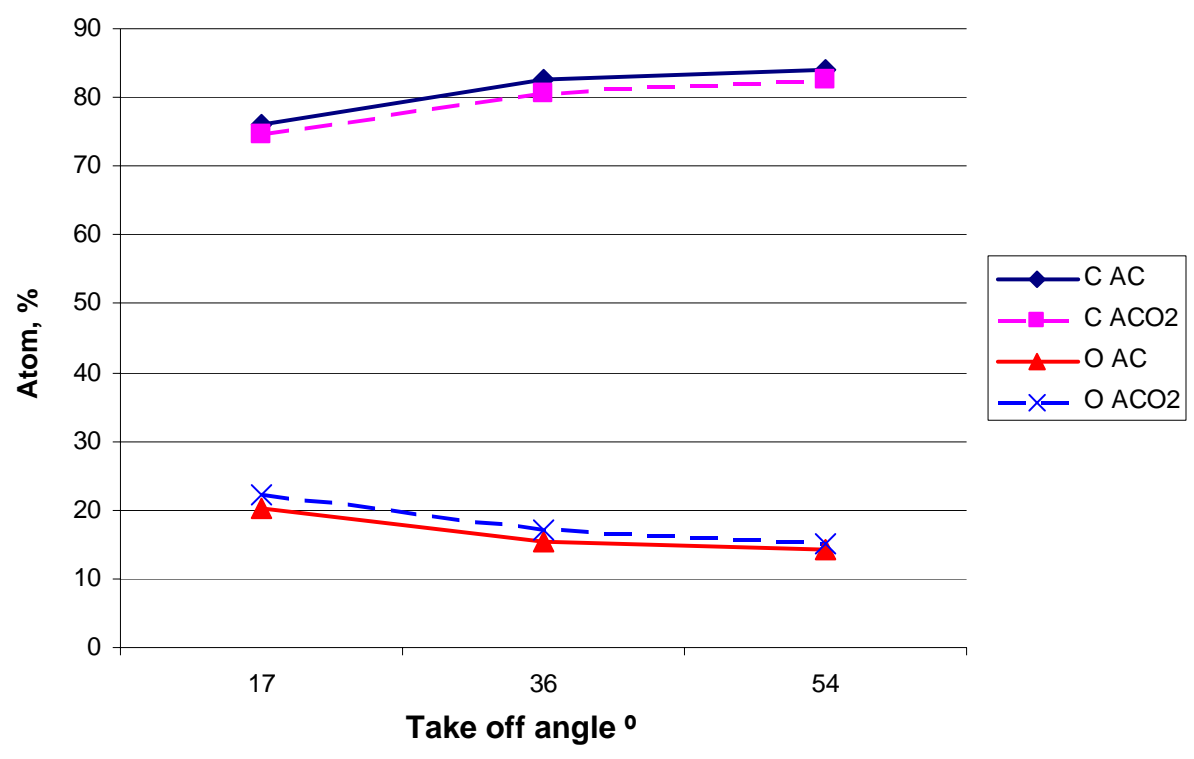

Fig. 6. Angle dependent carbon (C), oxygen (O) content of PP acetone films.

Conventional commercially available aldehyde functionalized glass slides (Genetix Limited) exhibit a functionalized surface thickness of $2.1 \mathrm{~nm}$ and a $\mathrm{C}=\mathrm{O}$ share of 2.8 $\%$ and are therefore comparable with the PP generated films. The major difference is the contact angle $\left(\mathrm{H}_{2} \mathrm{O}\right)$ of $55^{\circ}$ compared with the PP surfaces with values in the region of $40^{\circ}$ to $45^{\circ}$ which results in bigger spot sizes during the microarray analysis during the use of the last-mentioned.

The storage of the $\mathrm{C}=\mathrm{O}$ surfaces under inert atmosphere for $170 \mathrm{~h}$ at $50^{\circ} \mathrm{C}$ followed by XPS analysis proved to have no significant changes of the surface composition especially with respect to the amount of $\mathrm{C}=\mathrm{O}$ groups which are the predominant reaction partners for the immobilization of amino modified oligonucleotides.

Microarrays were performed on selected amino and carbonyl surfaces in order to give an initial useful lead as to whether PP films may be a tool for the generation of functionalized surfaces for this applications. The polymer support which was functionalized in the DEA1 series was tested with unmodified Cy5 labelled PCR products and 36mer oligonucleotides using different printing buffers. Addition of DMSO to the printing buffer improves the result of the experiment significantly. The capability of the PP amine film is proved in these initial experiments. 


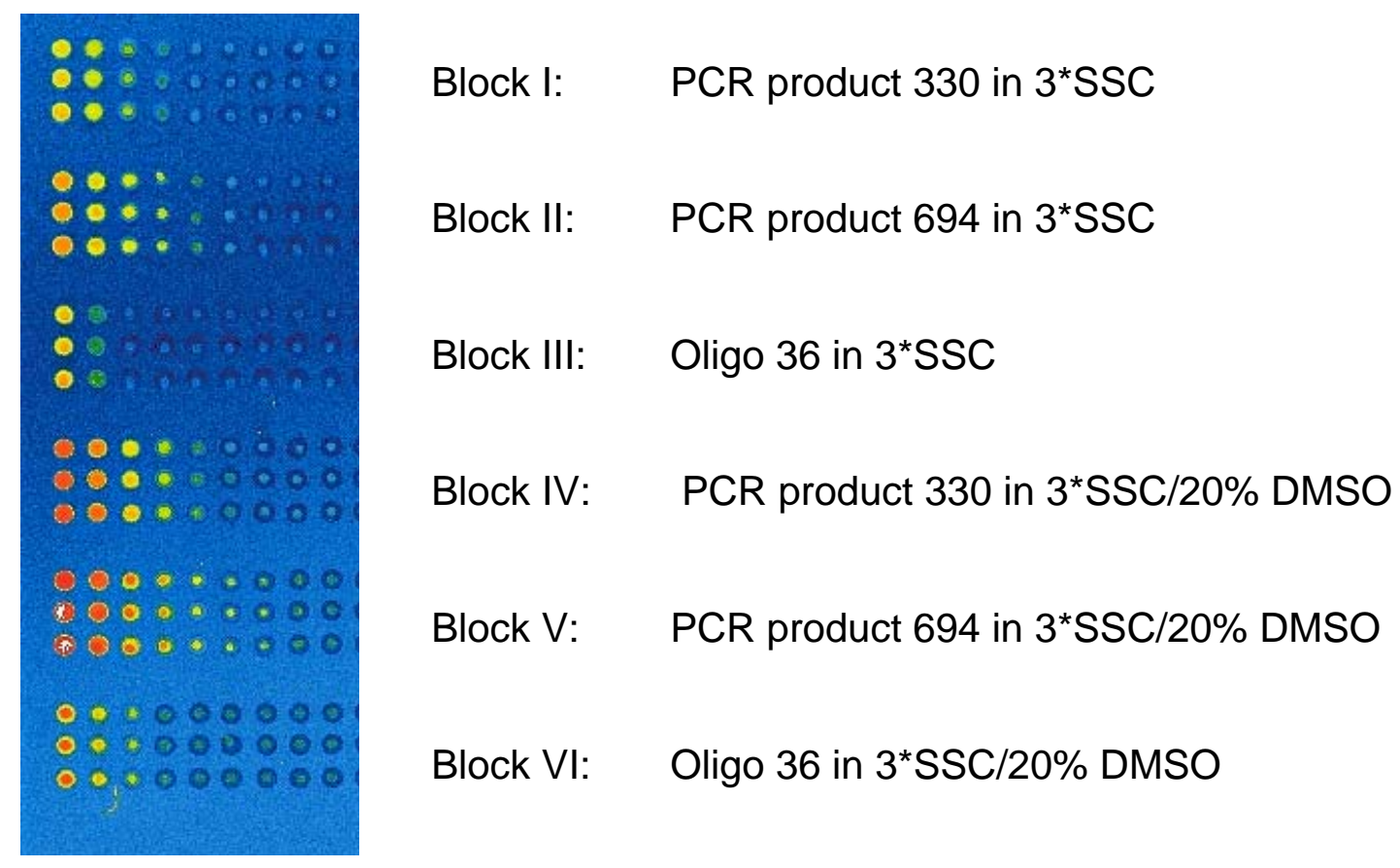

Fig. 7. Microarray on DEA1 series with Cy5 labelled PCR products and oligonucleotides.

While PP films exhibit higher amounts of nitrogen containing functional groups, the signal intensity is comparable with conventional sol gel based glass platforms indicating that the steric demand of the biomolecules are one limiting factor in the binding capacity. The improvement of the signal intensity with increasing size of the biomolecule 36mer $<330 \mathrm{mer}<694 \mathrm{mer}$ is in accordance with the results of standard protocols and a result of the ability of the biomolecule for ionic interaction with the surface as an initial step of the immobilization.

A covalent immobilization of amino modified oligonucleotides via PP carbonyl films is visualized in Fig. 8 via processing Cy3 as well as Cy5 labelled 23mers.

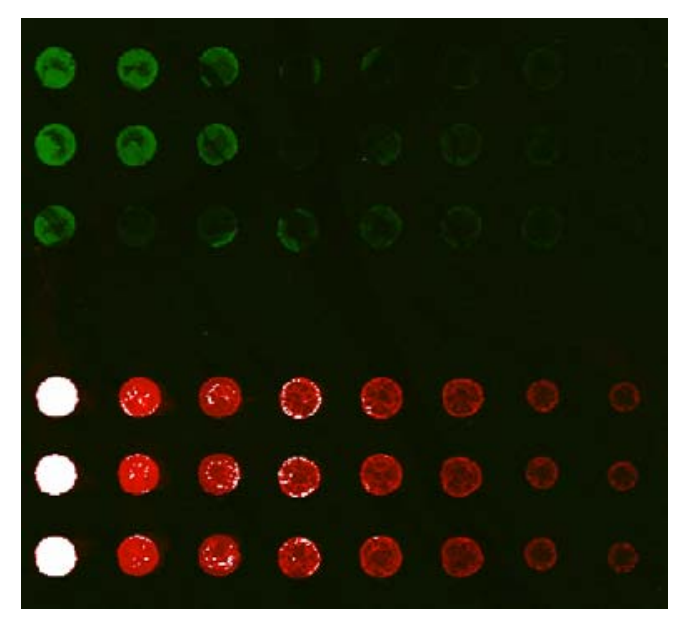

Fig. 8. Covalent immobilization of Cy5 and Cy3 oligonucleotides on the 03101 series. 
Dilution series printed in 3*SSC triplicates $(\mathrm{pmol} / \mu \mathrm{l}): 1 / 0.5 / 0.25 / 0.125 / 0.06$ / 0.03 / 0.015 / $0.007 / 0.004 / 0.002$. The performance of these first prototypes is satisfying with respect to the Cy5 signal intensity and the spot size. The Cy3 dye was partially destroyed during the $\mathrm{NaBH}_{4}$ reduction step and therefore this block could not be analyzed.

\section{Conclusions}

The plasma polymerization of several amines as well as acetone in the presence of argon as carrier gas yielded functional films with controlled amounts of amine and carbonyl functionalities. XPS methods have been used to get an overview of the thickness of the PP films and microarray experiments were able to prove the capability of the PP surfaces as platforms for novel applications such as microarray analysis. The next steps to optimize this pathway for microarray surfaces are the reduction of the background fluorescence of the PP films in the excitation range of 532 and $635 \mathrm{~nm}$ in order to improve the signal to noise ratios and finding of the optimum amine/carbonyl concentration to achieve the best possible binding capacity.

\section{Experimental section:}

\section{General procedure for Plasma Polymerization}

Diethylamine (>99 \%), acetone, ethylenediamine (>97\%), 1,4-diaminobutane, sodium cyanoborohydride, anhydrous ethanol and fluorescamin (98\%) were obtained by Sigma Aldrich $\mathrm{GmbH}$, Germany; argon 5.7 by the Messer Group $\mathrm{GmbH}$, Germany and $\mathrm{NH}_{3} 3.8$ by Linde $A G$, Germany. All monomers were used without preliminary purification steps, as received. All polymerization reactions were performed at reactor temperatures below $27{ }^{\circ} \mathrm{C}$ in reaction vessels $(32 * 50 * 60 \mathrm{~cm})$. The plasma was sustained by a $13.56 \mathrm{MHz}$ radio frequency signal generator and amplifier inductively coupled to the reactor vessel. The base pressure in the reactor was $0.01 \mathrm{mbar}$ (Edwards two stage E2M 275, EH 2600 mechanical booster). After reaching the base pressure the reactor was purged with argon for $10 \mathrm{~min}$ at a pressure of $0.02 \mathrm{mbar}$. Depending on the experimental setup different activation steps have been applied followed by evacuation to $0.01 \mathrm{mbar}$ and argon addition to 0.02 mbar. For all polymerizations a resulting pressure in the range of 0.015 up to 0.05 mbar was observed typically. After the plasma was switched off the monomer flow was maintained for 5 min followed by the evacuation of the reaction vessel for 30 min in order to minimise the post plasma oxygen uptake. Forthcoming experiments will provide data if this extensive post treatment results in significant differences compared with samples which are removed from the reactor immediately. Plasma polymers were deposited on cycloolefin based slides (Zeonor 1060 R, Zeon Corporation Japan) with a format of $25^{\star} 75^{\star} 1 \mathrm{~mm}$.

\section{Plasma Polymerization}

Diethylamine polymerization parameters type 1 (series DEA1): Activation: argon 150 sccm; $\mathrm{NH}_{3} 75 \mathrm{sccm}, \mathrm{p}=0.024 \mathrm{mbar}, 150 \mathrm{~W}, \mathrm{t}=5 \mathrm{~min}$. Polymerization: argon 50 sccm, diethylamine $=25 \mu \mathrm{l} / \mathrm{min}, \mathrm{p}=0.037 \mathrm{mbar}, 70 \mathrm{~W}, \mathrm{t}=20 \mathrm{~min}$.

Diethylamine polymerization parameters type 2 (series DEA2): Activation: argon 150 sccm; $p=0.016$ mbar, $180 \mathrm{~W}, \mathrm{t}=3 \mathrm{~min}$. Polymerization: argon $50 \mathrm{sccm}$, diethylamine $=25 \mu \mathrm{l} / \mathrm{min}, \mathrm{p}=0.026 \mathrm{mbar}, 95 \mathrm{~W}, \mathrm{t}=20 \mathrm{~min}$. 
Ethylenediamine polymerization parameters (series EDA): Activation: argon 150 sccm; $\mathrm{NH}_{3} 75 \mathrm{sccm}, \mathrm{p}=0.033 \mathrm{mbar}, 150 \mathrm{~W}, \mathrm{t}=5 \mathrm{~min}$. Polymerization: argon 50 $\mathrm{sccm}$, ethylenediamine $=20 \mu \mathrm{l} / \mathrm{min}, \mathrm{p}=0.028 \mathrm{mbar}, 80 \mathrm{~W}, \mathrm{t}=20 \mathrm{~min}$.

Acetone $/ \mathrm{O}_{2}$ polymerization (series $\mathrm{ACO} 2$ ): Activation: argon $210 \mathrm{sccm} ; \mathrm{O}_{2} 75 \mathrm{sccm}$, $\mathrm{p}=0.024$ mbar, $170 \mathrm{~W}, \mathrm{t}=5 \mathrm{~min}$. Polymerization: argon $50 \mathrm{sccm}, \mathrm{O}_{2} 75 \mathrm{sccm}$, acetone $=29 \mu \mathrm{l} / \mathrm{min}, \mathrm{p}=0.038 \mathrm{mbar}, 80 \mathrm{~W}, \mathrm{t}=20 \mathrm{~min}$.

Acetone polymerization (series AC): Activation: argon $210 \mathrm{sccm} ; \mathrm{O}_{2} 70 \mathrm{sccm}, \mathrm{p}=$ $0.023 \mathrm{mbar}, 180 \mathrm{~W}, \mathrm{t}=5 \mathrm{~min}$. Polymerization: argon $50 \mathrm{sccm}$, acetone $=45 \mu \mathrm{l} / \mathrm{min}, \mathrm{p}$ $=0.029 \mathrm{mbar}, 70 \mathrm{~W}, \mathrm{t}=20 \mathrm{~min}$.

X-ray photoelectron spectroscopy

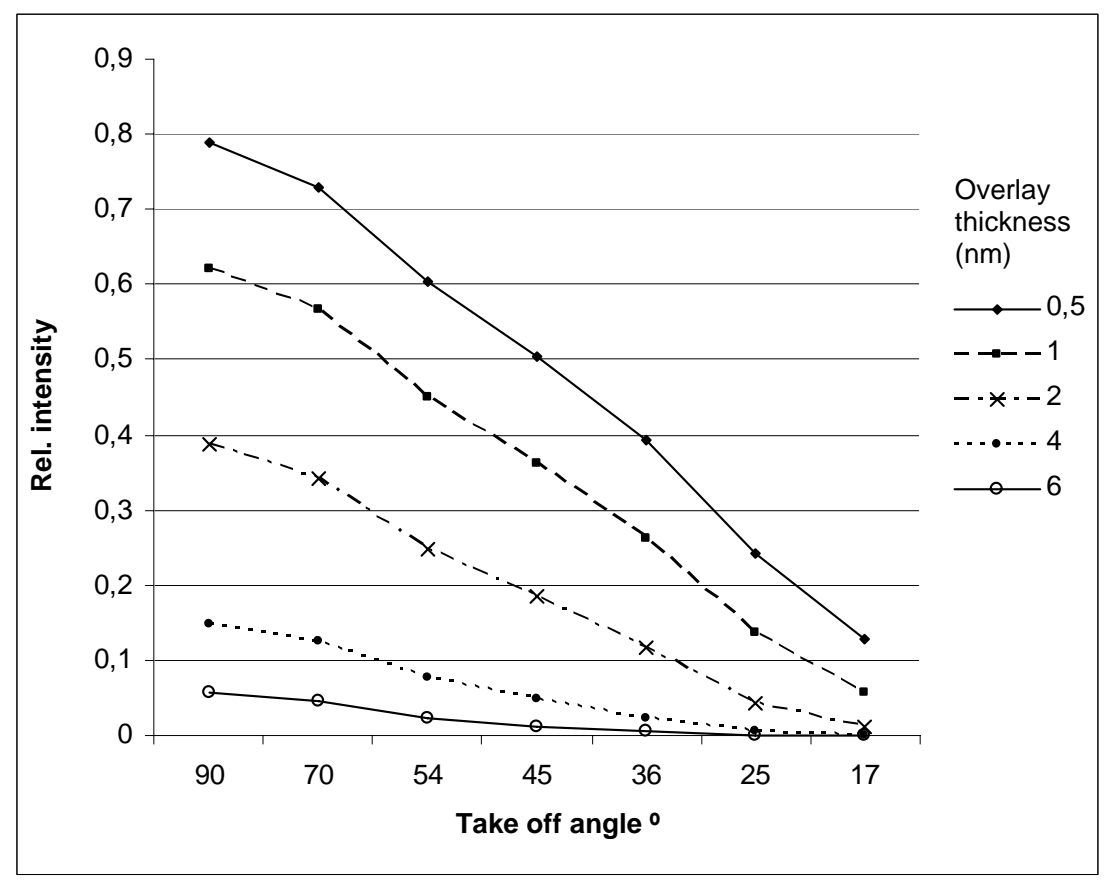

Fig. 9. Intensity as a function of the take off angle and the overlay thickness. Medium photoelectron path length is $2.1 \mathrm{~nm}$.

XPS was performed using a PHI 5500 Spectrometer equipped with a monochromatic Al-K $\alpha$ source. High resolution spectra have been obtained using a $\mathrm{Mg}-\mathrm{K} \alpha$ source. The take off angles between the surface and the electron optical axis was varied between 17 and $65^{\circ}$ in order to get detailed information of the overlay thickness of the different films (Fig. 9). The calibration of the spectrometer was done using the $\mathrm{Au}$ 4f $7 / 2$ peak position and the splitting between the $C$ 1s and the $F$ 1s peak positions of a PTFE (polytetrafluoroethylene) sample [19]. Following calculation has been used to be able to quantitatively compare the different experiments. Due to the fact that the intensity is a function of the take off angle and the overlay thickness, the share of the Intensity $I$ for selected angles can be used for the estimation of the layer thickness ( $I$ in $\%=(I x-I y) / I x ; I x=$ intensity at higher take off angles, $I y=$ intensity at lower take off angles):

$I=C \sin \theta e^{(d / \lambda \sin \theta)}$ 
where $I=$ intensity, $C=$ constant factor, $\theta=$ take off angle, $d=$ overlay thickness, $\lambda=$ medium path length of the photoelectrons in the overlayer.

\section{Contact angle goniometry}

Water contact angles were measured with the Top Drop Analyser 10 by Kruess, Germany using the sessile drop method. The averaged results obtained from six individual measurements of each sample were used.

\section{Microarray analysis}

Materials: Sodium-dodecyl-sulphate (SDS) $98 \%$ and 20 X SSC purchased from Promega, ethanol p.a., phosphate buffered saline, DMSO and $\mathrm{NaBH}_{4}$ from Sigma Aldrich $\mathrm{GmbH}$. The biometra UV crosslinker as well as the Axon 4000 a scanner with the GENE PIX PRO 4.0 software were used.

Ionic immobilization of fluorescent dye labelled PCR products and oligonucleotides on the amino surface DEA1

Dilution series printed in triplicates $(\mathrm{pmol} / \mu \mathrm{l}): 1 / 0.5 / 0.25 / 0.125 / 0.06 / 0.03$ / 0.015 / 0.007 / 0.004 / 0.002

\section{-Post Print process:}

$1000 \mathrm{ml} 4$ X SSC / $0.1 \%$ SDS (Sodium-dodecyl-sulphate) was prepared with $200 \mathrm{ml}$ 20xSSC, $10 \mathrm{ml} 10 \%$ SDS and $790 \mathrm{ml}$ deionized $\mathrm{H}_{2} \mathrm{O}$. Incubation of 15 min after the printing process (330 mer PCR product) was followed by the crosslinking (Stratalinker $0.120 \mathrm{~J}$ ), two times washing in $4 \times$ SSC / $0.1 \%$ SDS at room temperature and washing in $4 \times$ SSC / $0.1 \%$ SDS at $90{ }^{\circ} \mathrm{C}$. The slides were transferred into $70 \% \mathrm{ETOH}$ immediately. After drying, the printed slides were ready to be analyzed in conventional microarray scanners.

Covalent immobilization of fluorescent dye labelled aminomodified oligonucleotides on the ketone surface AC

The solution was prepared with $500 \mathrm{ml} \mathrm{NaBH}$ solution, $1.25 \mathrm{~g} \mathrm{NaBH}_{4}, 375$ 1x PBS and $125 \mathrm{ml} \mathrm{EtOH}$. Incubation over night after the printing process on the functionalized slide (33mer aminomodified oligonucleotide) was followed by $4 \mathrm{~min}$ washing each in $0.2 \%$ SDS solution, water at room temperature and water at $90{ }^{\circ} \mathrm{C}$ whilst shaking. After cooling to ambient temperature the slide was transferred into the $\mathrm{NaBH}_{4}$ solution for 5 min followed by washing for 2 min in $0.2 \%$ SDS solution and three times in deionized water. After drying, the printed slides were ready to be analyzed in conventional microarray scanners.

\section{References}

[1] Maskos, U.; Southern E. M., Nucleic Acids Res. 1992, 20, 1679.

[2] Shena, M.; Shalon, D.; Davis, R. W. Science 1995, 270, 467.

[3] Lipshutz, R. J.; Fodor, S. P.; Gingeras, T. R.; Lockhart, D. J. Nat. Genet. 1999, 21 (suppl.), 20.

[4] Adams, M. D.; Celniker, S. E.; Holt R. A. Science 2000, 287, 2185.

[5] Scherf, U.; Ross, D. T.; Waltham, M.; Smith, L. H.; Lee, J. K.; Tanabe, L.; Kohn, K. W.; Reinhold, W. C.; Myers, T. G.; Andrews, D. T.; Scudiero, D. A.; Eisen, M. B.; 
Sausville, E. A.; Pommier, Y.; Botstein, D.; Brown, P. O.; Weinstein, J. N. Nat. Genet. 2000, 3, 236.

[6] Kanoh, N.; Kumashiro, S.; Simizu, S.; Kondoh, Y.; Hatakeyama, S.; Tashiro, H.; Osada, H. Angew. Chem. 2003, 115, 5742.

[7] Riepl, M.; Schäferling, M.; Kruschina, M.; Ortigao, F.; Enander, K.; Liedberg, B. Langmuir 2002, 18, 7016.

[8] Angenendt, P.; Glokler, J.; Murphy, D. Analytical Biochemistry 2003, 309, 253.

[9] Afanassiev, V.; Hanemann, V.; Wolfl, S. Nucleic Acid Res. 2000, 28, E66.

[10] Arkles; B. Chemtech 1977, 7, 766.

[11] Ulbricht, M.; Riedel, M.; Marx, U. J. Membr. Sci. 1996, 120, 239.

[12] Shen, M. 'Plasma Chemistry of Polymers', Marcel Dekker Inc. 1976.

[13] Hollahan, J. R.; Bell, A. T. 'Techniques and Applications of Plasma Chemistry', John Wiley and Sons Inc. 1974.

[14] Haddow, D. B.; Steele, D. A.; Short, R.; Dawson, R. A.; Macneil, S. Journal of Biomedical Materials Research Part A 2003, 64A - 1, 80-87.

[15] Nitschke, M.; Uhlmann, S.; Schulze, R.; Werner, C. e-polymers 2006, no. 006.

[16] France, R. M.; Short, R. D.; Duval, E.; Jones, F. R. Chem. Mater. 1998, 10, 1176.

[17] Beamson, G.; Briggs, D. 'High-Resolution XPS of Organic Polymers: The Scienta ESCA300 Handbook', John Wiley and Sons Inc. 1992.

[18] Udenfriend, S. Science 1972, 178, 871.

[19] Blalock, E. 'A Guide to Microarrays', Kluwer Academic Publishers 2003, 10. 